\title{
In situ Synthesis of Pt-rare Earth Alloy Nanoparticles
}

\author{
$\underline{\text { Pei Liu }}{ }^{1}$, Yang $\mathrm{Hu}^{2}$ and Thomas Willum Hansen ${ }^{1}$
}

${ }^{1}$ DTU Nanolab, Technical University of Denmark, DK-2800, Kgs. Lyngby, Denmark.

${ }^{2}$ DTU Energy, Technical University of Denmark, DK-2800, Kgs. Lyngby, Denmark.

Platinum-rare earth metal (Pt-RE) alloys are superb electrocatalysts for a series of critical reactions for clean energy production. DFT calculations and experimental tests on bulk samples revealed that the high activity of Pt-RE systems originates from the compressively strained Pt overlayer on the alloy core, a structure generally formed in acidic working conditions. As a result, the d-band center of the Pt-rich surface is shifted to the optimum value, and the binding energies of reaction intermediates are optimized [1]. Compared to their Pt-late transition metal ( $\mathrm{Ni}$ or $\mathrm{Cu}$ ) alloy counterparts, Pt-RE alloys have lower dissolution potentials, and thus RE atoms on the particle surface are prone to leaching away resulting in the Pt-overlayer aforementioned. Besides, due to the large negative alloy formation energies, Pt-RE alloys exhibit great kinetic barriers for RE atom to diffusion from the particle core to the surface, which enable the alloy particles' exceptional stabilities in acidic media.

Scalable production of Pt-RE alloy nanoparticles via chemical synthesis route is very challenging.[2] Firstly, RE metals have very low standard reduction potentials, e.g., $-2.336 \mathrm{~V}$ for $\mathrm{Ce}^{3+} / \mathrm{Ce}$, which are very different from that of $\mathrm{Pt}^{2+} / \mathrm{Pt}(1.188 \mathrm{~V})$. As a result, it is not applicable to form Pt-RE alloys through a coreduction process of both metals. Secondly, RE metals are extremely oxyphilic. Presence of $\mathrm{O}_{2}$ and $\mathrm{H}_{2} \mathrm{O}$ must be avoided in the synthesis environment hampering scalable production. Thus far, only a few successful attempts have been reported, all of which used strictly controlled synthesis conditions such as ultrahigh vacuum and organic solvents.

Recently, Yang et al. developed an effective and versatile chemical route to produce Pt-RE alloys under moderate conditions [3]. Instead of directly reducing the metal salts in a $\mathrm{H}_{2} \mathrm{O}$ and $\mathrm{O}_{2}$ free environment, Yang's method requires only a simple heat treatment of a mixture of common precursors containing Pt, $\mathrm{RE}$, and a N-rich compound in a dilute $\mathrm{H}_{2}$ atmosphere. No glove box or strong alkaline solvents are needed. Moreover, this method is even capable of controlling the sizes and morphologies of the obtained Pt-RE alloy nanoparticles by tuning the synthesis conditions.

Regarding the alloy formation mechanisms via this synthesis approach, a great deal of crucial information is still missing. Particularly on the local crystalline structure and elemental evolution of the particles during the alloying process. In this work, we investigated the synthesis process of $\mathrm{Pt}_{5} \mathrm{Ce}$ alloy nanoparticles at atomic scale using in situ ETEM. The in situ synthesis experiments were performed on an FEI Titan 80-300 environmental transmission electron microscope (ETEM) equipped with a spherical aberration corrector (CEOS). The microscope was operated at $300 \mathrm{kV}$. A DENSsolutions wildfire heating chip was used to hold the precursors to proceed with the synthesis. Preliminary results (Figure 1) show $\mathrm{Pt}_{5} \mathrm{Ce}$ alloy nanoparticles were successfully formed in the ETEM in $2.6 \mathrm{mbar}_{2}$ at $550{ }^{\circ} \mathrm{C}$. The red arrow in Figure 1a denotes the (111) plane of pure Pt, while the yellow arrow in Figure 1b indicates the (100) plane of $\mathrm{Pt}_{5} \mathrm{Ce}$. Figure 2 shows the evolution of a $\mathrm{Pt}_{\mathrm{x}} \mathrm{Ce}$ alloy nanoparticle during the synthesis process. 


\section{References:}

[1] M Escudero-Escribano et al, Science 352, 6281 (2016), p. 73.

[2] VB Compton and BT Matthias, Acta Crystallogr. 12, 9 (1959), p. 651.

[3] Y Hu et al, J. Am. Chem. Soc.142, 2 (2020), p. 953.
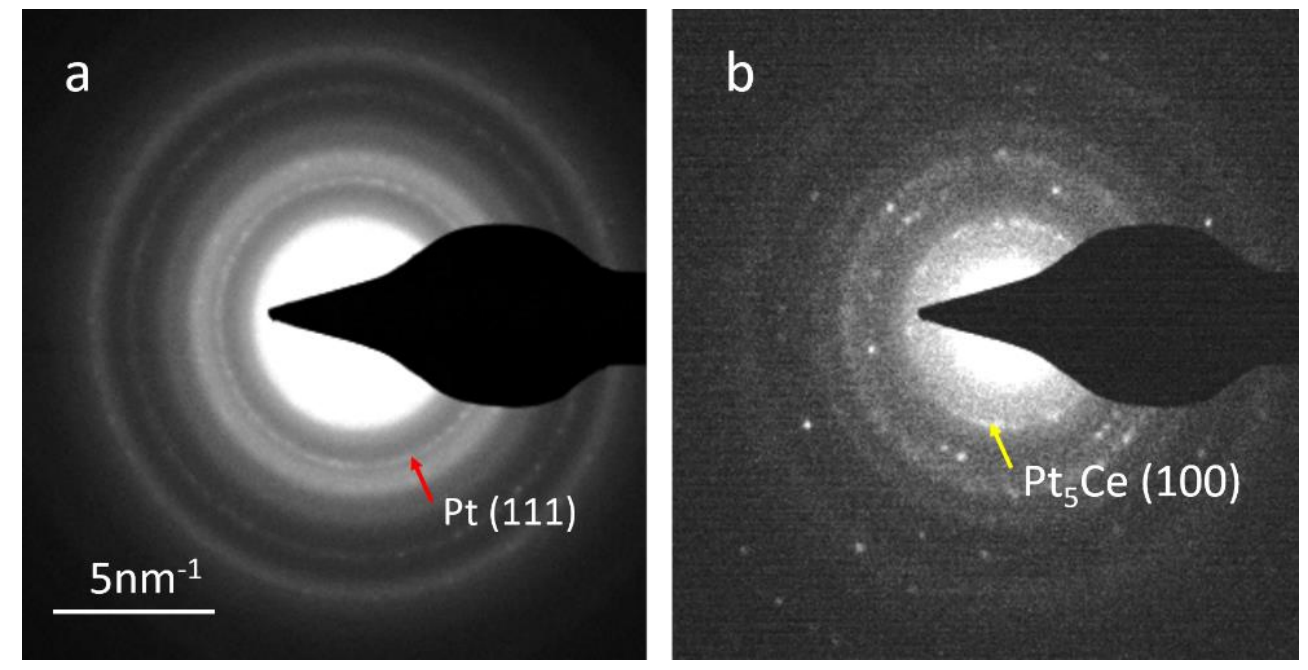

Figure 1. Diffraction micrographs before and after the synthesis process.
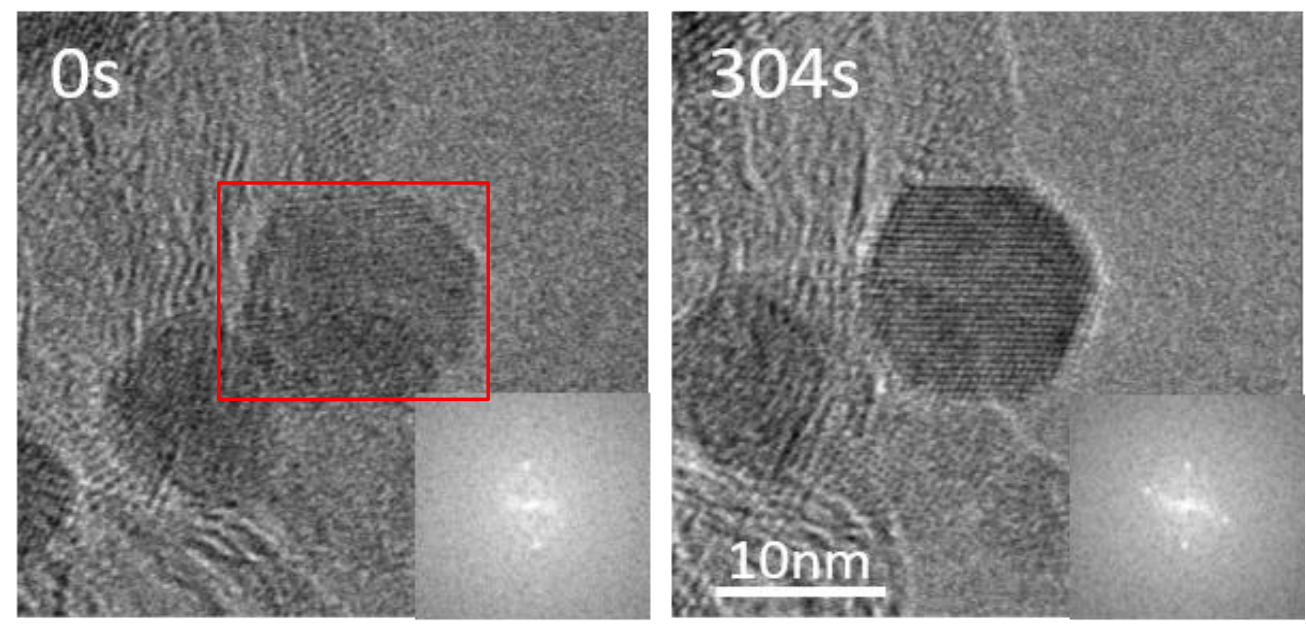

Figure 2. Morphology evolution of a $\mathrm{Pt}_{\mathrm{x}} \mathrm{Ce}$ nanoparticle. The HRTEM images were recorded using a Gatan OneView camera at an exposure time of $0.2 \mathrm{~s}$. 